\title{
ESTUDIO PRELIMINAR DE LA CAPACIDAD DE REMOCIÓN DE IONES INORGÁNICOS DE UNA ZEOLITA SINTÉTICA TIPO FAUJASITA
}

Fecha de recepción: 03 de marzo de 2015 • Fecha de evaluación: 15 de junio de 2015 • Fecha de aceptación: 21 de julio de 2015

\section{PRELIMINARY STUDY OF THE ABILITY OF INORGANIC IONS REMOTION BY SYNTHETIC ZEOLITE FAUJASITE TYPE}

Lis Manrique Losada ${ }^{1,5} \bullet$ Norma Constanza Bonilla² • Ricardo Chica Buitrago ${ }^{3} \bullet$ Jesús Hernando Otálora Bonilla ${ }^{3}$ Maurin Salamanca ${ }^{1,4}$

\section{RESUMEN}

La remoción de iones inorgánicos presentes en aguas residuales se realiza convencionalmente por adsorción e intercambio iónico. Las zeolitas, como la faujasita, poseen características físicas, químicas y estructurales que las hacen materiales potencialmente útiles como adsorbentes de contaminantes. Se sintetizó una zeolita tipo faujasita a partir de Al puro y sílica gel por el método sol-gel; se caracterizó por difracción de rayos X (DRX) y espectroscopía de infrarrojo (FTIR). Se determinó la capacidad de remoción de materia orgánica, sulfatos, fosfatos, nitrógeno amoniacal, dureza y alcalinidad presentes en aguas residuales domésticas (ARD) típicas de la ciudad de Florencia Caquetá y en aguas sintéticas. El trabajo experimental mostró buenos resultados a escala de laboratorio, con posible aplicación a escala real. Se identificó el material sintetizado como zeolita tipo faujasita Na-X. Debido al carácter básico de la zeolita sintetizada, solamente se observa remoción de cationes representados en dureza $\left(\mathrm{Ca}^{+2}\right.$ y $\left.\mathrm{Mg}^{+2}\right)$, nitrógeno amoniacal y materia orgánica (Demanda Química de Oxígeno, DQO). La remoción es debida a intercambio catiónico y a procesos de adsorción de material coloidal. La remoción de material contaminante en orden descendente es dureza $\left(\mathrm{Ca}^{+2}\right.$ y $\left.\mathrm{Mg}^{+2}\right)>\mathrm{NH}_{4}^{+}>$material coloidal (materia orgánica).

Palabras clave: Aguas residuales domésticas; Adsorción; Zeolita; Intercambio catiónico; Remoción de iones; Tratamiento de aguas residuales.

1 Grupo de Investigación Materiales, Ambiente y Desarrollo: MADE. Universidad de la Amazonia.

2 Grupo de Investigación GINMUA. Universidad de la Amazonia.

3 Grupo de Investigación en Materiales aplicados a desarrollos tecnológicos para la región y la enseñanza de la física. Universidad de la Amazonia.

4 Grupo de investigación en Química Ambiental y Tecnologías Limpias. Universidad Militar Nueva Granada.

5 Dirección de correspondencia: lismanrique@gmail.commailto:jesus_hernando_otalora_bonilla@hotmail.com 


\section{ABSTRACT}

The removal of inorganic ions present in wastewater is approached by physicochemical means such as adsorption and ion exchange. Zeolites such as faujasite have physical, chemical and structural characteristics that make them potentially useful materials for contaminant removal.

Faujasite-type zeolite from pure Al and silica gel by the sol-gel method was synthesized; The material was characterized by X-ray diffraction (XRD) and infrared spectroscopy (FTIR). The ability to remove organic matter, sulfates, phosphates, ammoniac nitrogen, hardness and alkalinity present in the Florencia (Caquetá) domestic wastewater and synthetic waters were determined. The experimental work showed good results on a laboratory scale and possible application on a larger scale. The synthesized material was identified as Na-X zeolite. Due to the basic character of the synthesized zeolite, it is observed the removal of hardness cations $\left(\mathrm{Ca}^{+2}\right.$ and $\mathrm{Mg}^{+2}$ ), ammonia nitrogen and organic matter (chemical oxygen demand, COD) is observed. The removal can be explained by the cation exchange and adsorption process of colloidal material. The removal of contaminating material in descending order is hardness $\left(\mathrm{Ca}^{+2} \mathrm{y} \mathrm{Mg}^{+2}\right)>\mathrm{NH}_{4}{ }^{+}>$colloidal material (organic matter).

Keywords: Domestic wastewater, Adsorption, Zeolite, Cationic interchange; lon removal; Wastewater treatment.

\section{INTRODUCCIÓN}

Las zeolitas son aluminosilicatos hidratados altamente cristalinos que al deshidratarse desarrollan, en el cristal ideal, una estructura porosa con diámetros de 3 a $10 \AA$ (microporos). Su estructura forma cavidades ocupadas por iones grandes y moléculas de agua con gran libertad de movimiento que permiten el intercambio iónico y la deshidratación reversible. Su estructura abierta les aporta gran capacidad para incorporar y ceder agua y cationes, sin cambios significativos en la estructura cristalina (Castaldi et al., 2008).

El proceso de intercambio iónico en las zeolitas está representado por la siguiente ecuación

$$
Z_{A} B^{Z+B}(\mathrm{z})+Z_{B} A^{Z+A}(s) \rightarrow Z_{A} B^{Z+B}(s)+Z_{B} A^{A+Z}(\mathrm{z})
$$

Donde $Z_{A}$ y $Z_{B}$ son las cargas de los cationes $A$ y $B, y$ los sufijos $z$ y s hacen referencia a la zeolita o a la solución, respectivamente. El comportamiento del intercambio iónico en las zeolitas depende de: la naturaleza de las especies catiónicas, el tamaño del catión tanto hidratado como deshidratado y la carga del catión, la temperatura, la concentración de las especies catiónicas en solución, las especies aniónicas asociadas al catión en solución, el solvente y las características de la zeolita (Rozic et al., 2000, Ríos et al., 2010).

Aunque las zeolitas son minerales de origen volcánico que pueden ser utilizadas en diversas aplicaciones; también pueden ser sintetizadas en laboratorio. Se ha realizado la síntesis de zeolitas utilizando fuentes no convencionales, como el trabajo desarrollado por Dussan et al. (2010), donde se preparó una zeolita tipo faujasita a partir de cuarzo y aluminio grado analítico. Los autores confirmaron la obtención de la estructura propuesta utilizando diferentes técnicas de caracterización tales como microscopia de fuerza atómica (AFM), microscopía electrónica de barrido 
(SEM), difracción de rayos-x (XRD) y medidas de espectroscopia de energía dispersiva de rayos X (EDX).

Algunas de las aplicaciones de las zeolitas, tanto naturales como sintéticas, son la filtración de gases nocivos para el entorno hasta el mejoramiento de suelos, nutrición animal o aditivo al cemento para elaborar hormigones aligerados destinados a la construcción (Grogg, 2010).

Las zeolitas también han sido utilizadas en el tratamiento de aguas residuales, ya que constituyen el mejor sistema de filtración natural existente, ofrecen un efecto superior a la arena o los filtros de carbón, resultando en agua más pura con mejor productividad y requiriendo menos mantenimiento. La estructura altamente porosa de las zeolitas puede capturar partículas contaminantes de hasta $4 \mu \mathrm{m}$ y si se encuentran cargadas negativamente de forma natural, pueden adsorber cationes, como metales pesados y amonio, así como también algunos contaminantes orgánicos que generan olores no deseados (Nibou et al., 2010).

La capacidad de las zeolitas para reducir la solubilidad de metales pesados a través de la inmovilización química es de particular interés (Castaldi et al., 2008). El proceso se basa en el intercambio iónico entre metales pesados y cationes, tales como el sodio o potasio, o en la precipitación de hidróxidos metálicos sobre la superficie externa de la zeolita. El proceso de intercambio iónico en la zeolita es influenciado por varios factores como la concentración y naturaleza de los cationes y aniones, el valor del $\mathrm{pH}$ y la estructura cristalina de la zeolita (Sprynskyy et al., 2006). La zeolita natural clinoptilolita fue utilizada para la adsorción de metales pesados, demostrando mayor selectividad para el catión $\mathrm{Zn}^{+2}$. La interacción entre este metal, el cual tiene el radio iónico más pequeño, y la superficie activa de la zeolita, fue más fuerte respecto a la interacción con $\mathrm{Pb}^{+2}$ y $\mathrm{Cd}^{+2}$. Se demostró que los metales se adsorbieron no solo en la superficie de la zeolita sino también en las jaulas y los canales de la estructura porosa (Castaldi et al.,
2008). Se ha demostrado que zeolitas sintéticas como la NaX y la NaHX pueden ser usadas como intercambiadores iónicos o adsorbentes para recuperar iones $\mathrm{Fe}^{+3}$ de suelos y aguas (Nibou et al., 2010). De la misma forma la zeolita $\mathrm{NaA}$ resultó muy efectiva en la remoción de $\mathrm{Zn}^{2+}$ (Nibou et al., 2010b). Se ha reportado el tratamiento de soluciones acuosas contaminadas con iones $\mathrm{Ni}^{2+}, \mathrm{Pb}^{2+}, \mathrm{Zn}^{2+}, \mathrm{Cr}^{6+}, \mathrm{Cd}^{2+}$ y $\mathrm{Co}^{2+}$ a través de intercambio iónico utilizando zeolita faujasita $\mathrm{Na}$ $\mathrm{Y}$, demostrando que la afinidad de la zeolita $\mathrm{Na}-\mathrm{Y}$ por los iones estudiados varía así: $\mathrm{Co}^{2+}>\mathrm{Ni}^{2+}>\mathrm{Zn}^{2+}>$ $\mathrm{Pb}^{2+}>\mathrm{Cr}^{6+}>\mathrm{Cd}^{2+}$ (Mekatel et al., 2012).

El creciente interés por el ambiente, hace necesario implementar tratamientos eficaces que eviten el deterioro de la calidad de las aguas, especialmente por el vertido de efluentes domiciliarios. El agua residual doméstica (ARD), contiene una alta cantidad de contaminantes, como sales metálicas con cationes $\left(\mathrm{Ca}^{2+}, \mathrm{Mg}^{2+}, \mathrm{Ba}^{2+}, \mathrm{Pb}^{+2}, \mathrm{Fe}^{+2}, \mathrm{Fe}^{+3}\right)$ y aniones $\left(\mathrm{F}^{-}, \mathrm{NO}_{3}\right.$, $\mathrm{AsO}_{4}{ }^{3-}, \mathrm{CrO}_{4}{ }^{2-}, \mathrm{SeO}_{4}{ }^{2-}$ ), los cuales son perjudiciales para los ecosistemas acuáticos. (Fu y Wang, 2011)

La quebrada La Perdiz atraviesa la ciudad de Florencia (Caquetá) en dirección nororiente - suroccidente, en un recorrido de aproximadamente 3,76 $\mathrm{km}$, y se ha convertido en el receptor principal de las ARD del municipio, lo que ha ocasionado el desequilibrio de este ecosistema acuático, afectando la calidad de sus aguas.

Los altos contenidos de coliformes totales y E.coli, en el tramo urbano de la quebrada, permiten clasificar el agua como no apta para consumo doméstico o para uso recreativo de contacto primario o secundario (Manrique y Peláez, 2010). Así mismo, la quebrada La Perdiz se encuentra afectada por la intervención antrópica en las riberas a su paso por la ciudad. El vertimiento directo de ARD, hospitalarias e industriales sobre la Quebrada La Perdiz contribuye al deterioro de la calidad del agua del ecosistema en cuestión, al detrimento de su vegetación riparia, caudal y metabolismo. Además, de generar irregularidades de tipo 
ambiental y de salubridad a niveles preocupantes, perdiendo, con esto, el municipio de Florencia y el departamento del Caquetá, una fuente hídrica de incalculable valor para su desarrollo económico y social.

De acuerdo a lo anterior, se plantea la necesidad de implementar estrategias que contribuyan con el mejoramiento de este ecosistema acuático con el propósito de que pueda ser apto para uso agrario, pecuario, entre otros. Una alternativa para suplir esta necesidad, radica en el tratamiento de las ARD que se descargan a la quebrada debido al sistema de alcantarillado o a descargas puntuales, dado que estas son las que generan la problemática. Estos resultados constituyen el primer paso en la búsqueda de materiales alternativos de bajo costo con múltiples aplicaciones, tales como, la solución de problemas regionales, como lo es la remoción de contaminantes en aguas residuales.

Aprovechando la capacidad de intercambio iónico y de adsorción de las zeolitas, así como, la posibilidad de su obtención a escala de laboratorio con materiales de bajo costo, se seleccionó una zeolita tipo faujasita sintética para estudiar la capacidad de remoción de contaminantes, presentes en ARD que son descargadas en la Quebrada la Perdiz. En este artículo, se presentan los resultados preliminares de la caracterización de la zeolita y su aplicación al tratamiento de $A R D$, en términos de remoción de iones presentes en el agua. Se implementaron procesos de adsorción en baterías experimentales de laboratorio.

\section{MATERIALES Y MÉTODOS}

\section{Preparación de la zeolita}

La zeolita se preparó a partir de Al grado analítico (Carlo Erba) y silica gel, por el método sol-gel modificado (Dussan et al, 2010), a partir de geles de $\mathrm{Al}_{2} \mathrm{O}_{3}$, $\mathrm{SiO}_{2}$ y $\mathrm{H}_{2} \mathrm{O}$. A temperatura ambiente se mezcló una solución de $\mathrm{NaOH} 1.8 \mathrm{M}$ con $6 \mathrm{~g}$ de $\mathrm{SiO}_{2}$, lentamente, hasta completar una dispersión homogénea, bajo agitación continua. De manera simultánea se preparó una solución de $\mathrm{NaOH} 5.3 \mathrm{M}$ la cual se adicionó lentamente y bajo agitación constante a 1,7 g de Al. Una vez se obtuvieron ambas disoluciones se adicionó la solución que contenía aluminio a la que contenía óxido de silicio, con agitación continua, para evitar la formación de aglomerados. El gel resultante, que era altamente viscoso, se hizo reaccionar a condiciones hidrotérmicas, en un autoclave de acero inoxidable a 120 durante $10 \mathrm{~h}$. Los productos fueron recuperados por centrifugación para precipitar la zeolita. Se realizó el lavado de la zeolita con agua destilada hasta $\mathrm{pH}$ neutro. Posteriormente fue secada a $100^{\circ} \mathrm{C}$ durante $10 \mathrm{~h}$.

\section{Caracterización de la zeolita}

El análisis de infrarrojo fue llevado a cabo utilizando el método de pastillas de $\mathrm{KBr}$ en un espectrofotómetro Nicolet Manga IR560 con un detector $\mathrm{MCT} / \mathrm{A}$ a $-196^{\circ} \mathrm{C}$, entre 600 y $4000 \mathrm{~cm}^{-1}$. La difracción de rayos $X$ se realizó en un equipo Rigaku modelo Miniflex con fuente de $\mathrm{Cu}(\boldsymbol{\lambda}=1,5418 \AA)$ operada a $40 \mathrm{kV}$ y $30 \mathrm{~mA}$ para valores de 3 a $65^{\circ}$ a una velocidad de barrido de $2 \%$ min.

Toma de muestras y caracterización del agua residual

El sitio para la toma de muestras de aguas residuales a analizar, se encontraba en una descarga de un box coulvert ubicado en el barrio el Raicero. Este sitio se consideró representativo porque colecta, en un $90 \%$, aguas residuales domésticas provenientes de los barrios del noroeste de la ciudad.

Las muestras se colectaron en recipientes de polietileno con tapa hermética y se transportaron refrigeradas al laboratorio para su análisis inmediato. Se siguieron las técnicas de muestreo y preservación de muestras planteadas por el IDEAM (Código: TI0187). Para caraterizar las ARD se determinó la Demanda Química de Oxígeno (DQO) por el método de reflujo cerrado, fósforo reactivo por el método del ácido ascórbico, sulfatos por el método turbidimétrico, 
dureza total (Mg y Ca) por método titulométrico y nitrógeno amoniacal por el método de Nessler. Todos los análisis se realizaron de acuerdo con los métodos estándar para análisis de aguas residuales, propuestos por APHA-AWWA-WPCF.

\section{Estudios de adsorción}

Para determinar la capacidad de adsorción de la zeolita se plantearon dos baterías experimentales utilizando dos tipos de agua; un tipo de ARD, que llamaremos sintética, compuesta por una solución que contenía iones $\left(\mathrm{Ca}^{+2}\right)$ y un segundo tipo era ARD real. Se evaluó si la capacidad de adsorción o intercambio iónico se ve afectada por la presencia de otros iones en el agua y si existe un efecto competitivo entre contaminantes. Las baterías experimentales consistieron en colocar $100 \mathrm{~mL}$ del agua problema a adsorción durante 90 minutos con agitación constante, a diferentes concentraciones de zeolita $\left(0,1-0,8 \mathrm{~g} \cdot \mathrm{L}^{-1}\right)$ y de ARD inicial, sintética y real (Tabla 1). Después del tiempo de adsorción se determinó la concentración final de iones (dureza, $\mathrm{NH}_{4}^{+}$) y de materia orgánica (DQO) por los métodos anteriormente mencionados.

La capacidad de adsorción, se presenta por medio del porcentaje de remoción calculado según la ecuación (1):

$$
\text { \% Remoción }=\left(\left(C_{i n}-C_{f i n}\right) / C_{i n}\right) \times 100
$$

Donde $C_{\text {in }}$ es la concentración inicial de un ion en la solución, $C_{\text {fin }}$ es la concentración final del ion después de la adsorción.
Posteriormente, se desarrollaron experimentos en una columna de adsorción de borosilicato de $2,9 \mathrm{~cm}$ de diámetro. Se trabajaron 3 alturas de lecho de zeolita y se midió la concentración de los iones en el efluente después de una hora de adsorción. A partir de las curvas de quiebre para cada caso, se determinó la capacidad de adsorción del lecho $q_{b}$ con la ecuación (2)

$$
q_{b}=\frac{Q_{v} \times t_{10 \%} \times C_{o}}{m_{c}}
$$

Donde: $q_{b}$ es la cantidad de soluto adsorbido a la ruptura (mg/g adsorbente), $t_{10 \%}$ es el tiempo de servicio (min) obtenido cuando la concentración del efluente alcanzó el 10\% de la concentración del afluente, $C_{0}$ es la concentración de ion metálico en el afluente (mg. $\left.\mathrm{L}^{-1}\right), \mathrm{Q}_{v}$ es el flujo volumétrico del efluente $\left(\mathrm{L} \cdot \mathrm{min}^{-1}\right)$ y $\mathrm{m}_{\mathrm{c}}$ es la masa de adsorbente $(\mathrm{g})$.

\section{RESULTADOS Y DISCUSIÓN}

\section{Caracterización zeolita sintetizada}

La Figura 1 muestra el espectro infrarrojo de la zeolita sintetizada. Las señales principales de este tipo de materiales se encuentran en el intervalo en número de onda de $4000-800 \mathrm{~cm}^{-1}$. Se puede observar la banda entre 1100 a $950 \mathrm{~cm}^{-1}$ pertenece a la especie Si-O-Al (Dussan et al., 2012). Aproximadamente a $1000 \mathrm{~cm}^{-1}$ se encuentran las bandas correspondientes a las vibraciones asimétricas del estiramiento del enlace T-O (T=Si o Al). Por otro lado, la banda entre 3750 hasta $3450 \mathrm{~cm}^{-1}$ es atribuida a

\begin{tabular}{|c|c|c|}
\hline Tipo de ARD & lon inorgánico & Concentración Inicial (mg.t-1) \\
\hline Sintética & $\mathrm{Ca}^{2+}$ (dureza) & 240,140 y 86,4 \\
\hline Real & Nitrógeno amoniacal $\left(\mathrm{NH}^{+4}\right)$, dureza y DQO & $\begin{array}{l}\text { DQO para ARD alta: } 424 \\
\text { DQO para ARD media: } 284 \\
\text { DQO para ARD baja: } 144\end{array}$ \\
\hline
\end{tabular}

Tabla 1. Concentraciones iniciales de las ARD sintética y real 
Si-OH y grupos hidroxilo. Las bandas en la región $3400-3600 \mathrm{~cm}^{-1}$ están asociadas a vibraciones $\mathrm{O}-\mathrm{H}$ de agua en interacción por $\mathrm{H}$-enlazante con un átomo de oxígeno. La banda a $3689 \mathrm{~cm}^{-1}$ correspondería a agua coordinada con las cargas que compensan iones alcalinos. La presencia de agua no disociada es confirmada por la detección de una banda débil a $1600 \mathrm{~cm}^{-1}$, la cual es característica de vibraciones para agua (HOH) (Moulin et al., 2008). El agua siempre se detectará sobre la superficie de esta zeolita básica aunque presente una extensión muy pequeña (Moulin et al, 2008). La banda en aproximadamente $1600 \mathrm{~cm}^{-1}$ muestra que no se ha llevado a cabo una deshidratación completa en los productos finales de la síntesis $\left(\mathrm{H}_{2} \mathrm{O}\right.$ deform ation mode).

La figura 2 presenta los difractogramas para la zeolita sintetizada en este trabajo y para la zeolita $X$ (ICSD \# 6315) tipo faujasita, a partir de los difractogramas se encuentra un predominio de la fase cristalina. Se puede decir que la zeolita sintetizada en el laboratorio corresponde a una zeolita Faujasita $\mathrm{Na}$-X.

\section{Capacidad de remoción de iones individuales en agua sintética}

Teniendo en cuenta que la zeolita tiene un carácter básico, su capacidad de intercambio y adsorción se presenta sobre cationes. Se hizo seguimiento a la concentración de cationes $\mathrm{Ca}^{+2}$ y $\mathrm{Mg}^{+2}$ en solución, con una dureza de 240, 140 y 86,4 mg. $\mathrm{L}^{-1}$. El incremento en la dosis de zeolita mejora la capacidad de remoción de dureza hasta llegar al 100\% con una dosis de 0,4 g.L-1 en todas las soluciones (concentración alta, media y baja) (Fig. 3). El Análisis de Varianza realizado a los resultados de remoción de dureza, con los factores: dureza inicial y dosis de zeolita, indica una correlación estadísticamente significativa $(p<0,05)$ entre la dosis de zeolita y el porcentaje de remoción de dureza, con un coeficiente de correlación de 0,9325. La dureza inicial no afecta el porcentaje de remoción, no hay correlación significativa.

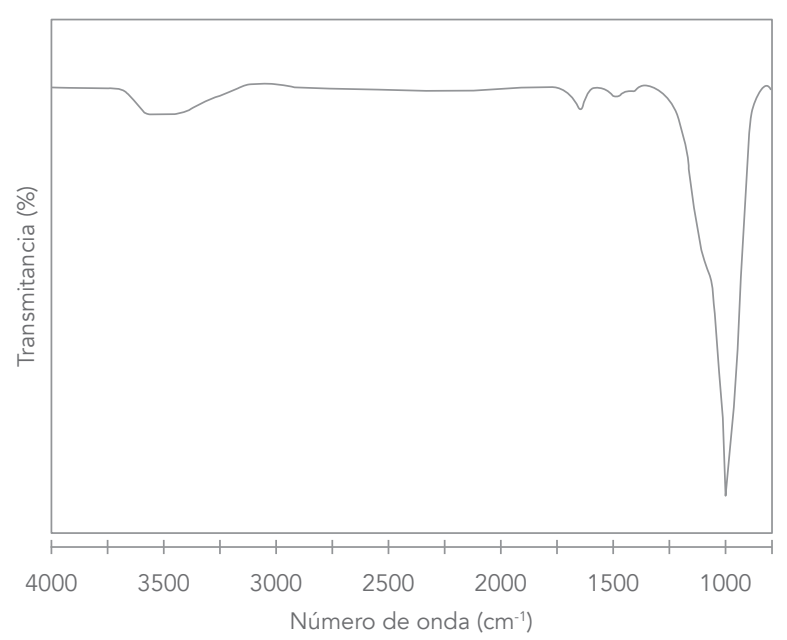

Figura 1. Espectro IR de la zeolita Faujasita sintetizada

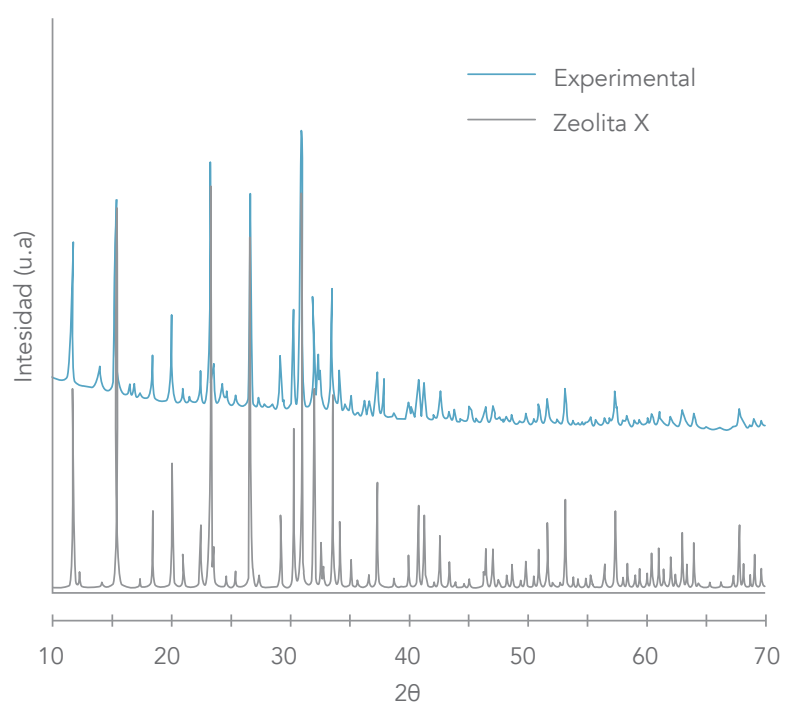

Figure 2. Comparación DRX: zeolita Faujasita sintética de este trabajo y zeolita $\mathrm{Na}-\mathrm{X}$.

\section{Capacidad de remoción de iones en ARD}

Los resultados de las pruebas de adsorción se presentan en términos de porcentajes de remoción de cada ion. Se trabajaron tres tipos de aguas, con concentración alta, media y baja de materia orgánica (DQO). Se observó que al aplicar adsorción, la remoción de los aniones era nula, es decir que no 
se presentó remoción de fosfatos, nitratos, nitritos y alcalinidad. El carácter básico de esta zeolita implica adsorción entre poros (jaulas y ventanas) del adsorbente e intercambio catiónico.

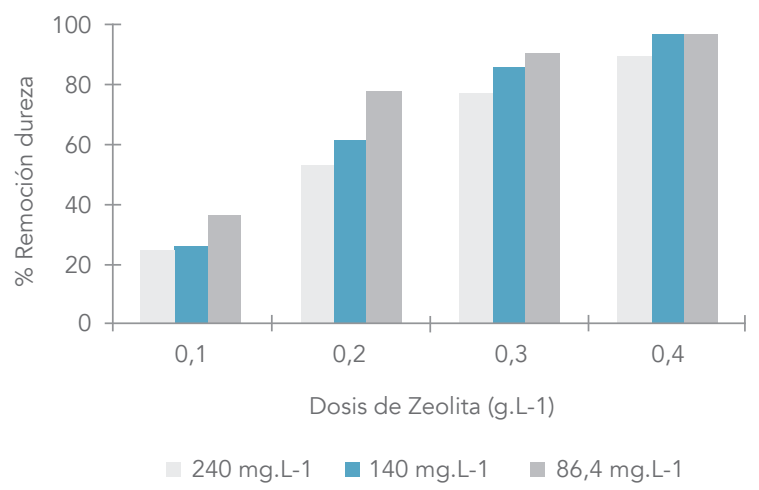

Figura 3. Remoción de dureza en agua sintética a diferentes dosis de zeolita.

El nitrógeno amoniacal es una forma iónica de nitrógeno en solución acuosa. Este contaminante proviene principalmente de ARD, fertilizantes, AR industriales y residuos agrícolas. El nitrógeno amoniacal contribuye a acelerar el proceso de eutroficación de ríos, al descenso del oxígeno disuelto y es un tóxico para organismos acuáticos. El nitrógeno amoniacal, está presente en ARD, principalmente cuando es reciente la contaminación de la misma, y por tanto es una evidencia de alto contenido de materia principalmente orgánica proveniente de los residuos mencionados (Roldan y Ramírez, 2008, Manrique y Peláez, 2010).

La Figura 4 presenta los porcentajes de remoción de nitrógeno amoniacal alcanzados bajo diferentes dosis de zeolita y con diferentes concentraciones iniciales de este ion. Esta especie iónica fue removida hasta el $100 \%$ en un ARD baja, utilizando un sistema en discontinuo con una dosis baja de zeolita $\left(0,1 \mathrm{~g} \cdot \mathrm{L}^{-1}\right)$. En el caso de aguas con alta concentración de nitrógeno amoniacal (ARD en época de bajas lluvias), se logran remociones hasta del $50 \%$ de este ion con dosis mayores a 0,4 g.L-1 de zeolita. Este comportamiento también se observa para concentraciones intermedias. Para dosis mayores a 0,4 g.L.-1 no se observan incrementos en los niveles de remoción. A mayor concentración de $\mathrm{NH}_{4}{ }^{+}$en el ARD cruda, menor es la capacidad de remoción, esto puede atribuirse, en ese caso, a que los sitios de intercambio de la zeolita no serán suficientes. En estudios previos se ha revelado que se alcanza la mayor remoción de éste ion cuando los sitios de intercambio están con la especie sodio (Wang y Peng, 2010). Rozic et al (2000) obtuvieron para la clinoptilolita, que las mayores remociones de nitrógeno amoniacal se alcanzaron a concentraciones iniciales bajas, con el incremento de la concentración inicial, la eficiencia disminuye rápidamente. Esto se puede explicar por el tamaño del nitrógeno amoniacal que facilita el intercambio iónico con los cationes sodio de la zeolita. La capacidad de adsorción solo sería posible en la superficie si el tamaño de poro fuera igual o menor al tamaño del ion amoniaco.

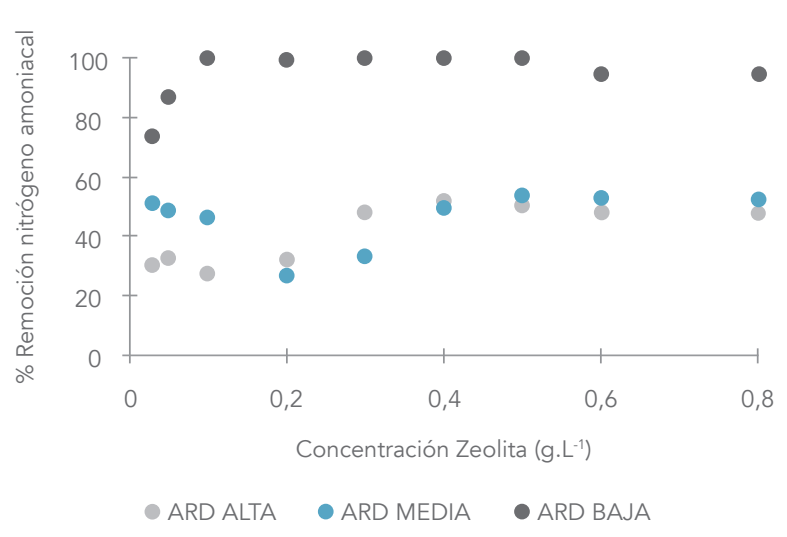

Figura 4. Porcentaje de Remoción de Nitrógeno amoniacal.

Se observó una alta afinidad entre los cationes $\mathrm{Ca}^{+2}$ y $\mathrm{Mg}^{+2}$ y los poros de la zeolita Faujasita básica, pues sin importar la concentración de dureza, se alcanzan porcentajes de remoción entre el 90 y 100\%, para dosis entre 0,4 y 0,5 g.L.-1 (Fig. 5). Este comportamiento está de acuerdo con lo reportado por Wang y Peng (2010). 
Los iones $\mathrm{Ca}^{+2} \mathrm{y} \mathrm{Mg}^{+2}$ son removidos principalmente por intercambio catiónico con el $\mathrm{Na}^{+}$de la zeolita al igual que para el nitrógeno amoniacal. Para un sistema multicomponente trabajado en la literatura con la zeolita natural clinoptilolita, se encontró que había un efecto competitivo entre el amonio y los cationes $\mathrm{Ca}^{+2}$ y $\mathrm{Mg}^{+2}$ y que la selectividad de la zeolita para iones amonio era predominante (Wang y Peng, 2010). Sin embargo, la faujasita de éste trabajo presenta el comportamiento contrario, presenta alta selectividad hacia los cationes que generan dureza.

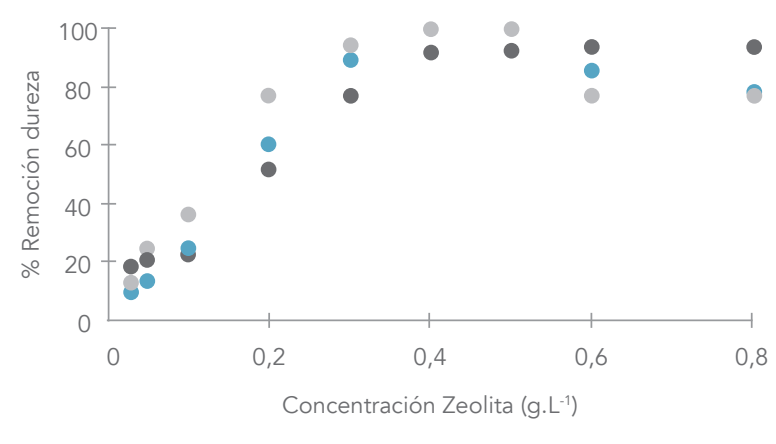

- ARD ALTA - ARD MEDIA - ARD BAJA

Figura 5. Porcentaje de Remoción de Dureza $\left(\mathrm{Ca}^{+2} \mathrm{y} \mathrm{Mg}^{+2}\right)$.

La remoción de materia orgánica se favorece cuando su concentración inicial en el ARD es baja (144 mg. $\left.\mathrm{L}^{-1}\right)$, donde se logran remociones hasta del $85 \%$ con dosis de zeolita de 0,3 g.L-1 (Fig. 6). Cuando se trata de concentraciones altas de ARD típica de la ciudad (424 mg. $\left.\mathrm{L}^{-1}\right)$, el porcentaje de remoción no supera el $63 \%$ para una dosis de $0,4 \mathrm{~g} \cdot \mathrm{L}^{-1}$ de zeolita. Para concentraciones medias de ARD, los porcentajes de remoción son bajos; solamente con dosis bajas $\left(0,05 \mathrm{~g}^{\left.-\mathrm{L}^{-1}\right)}\right.$ se alcanzan remociones del $38 \%$.

La materia orgánica (coloidal) con carga superficial catiónica entra en competencia por sitios de intercambio con los otros cationes presentes en el ARD. Con el

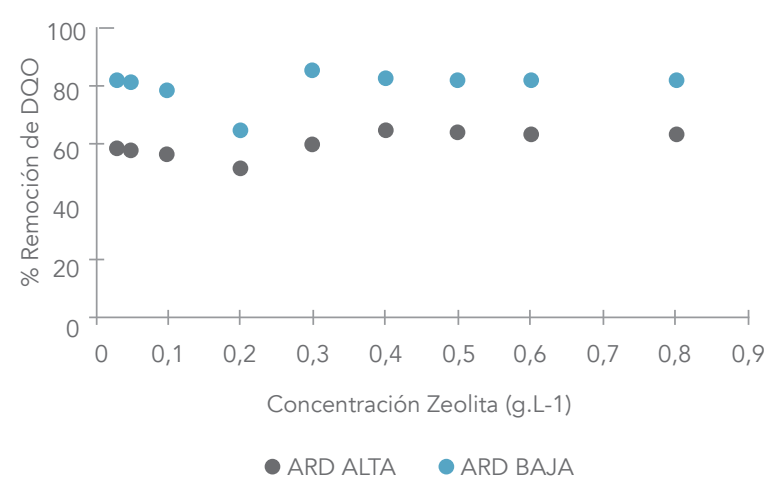

Figura 6. Porcentaje de Remoción de Materia Orgánica (DQO).

incremento de la $\mathrm{DQO}$ en el ARD, también aumentan los cationes que tienen mayor afinidad con los sitios de intercambio y de adsorción, de ésta forma la remoción de materia orgánica se desfavorece. Se observó que la afinidad de la zeolita $\mathrm{Na}-\mathrm{X}$ para los iones estudiados varía de la siguiente forma: $\mathrm{Ca}^{+2}, \mathrm{Mg}^{+2}>$ $\mathrm{N}-\mathrm{NH}^{+4}>$ Materia Orgánica. Estos resultados pueden ser explicados por las propiedades físicas y químicas de los iones metálicos así como de sus radios iónicos (Hadj et al, 2012). Éste último es un factor importante para el intercambio en términos de difusión y el sitio de adsorción en la estructura de la zeolita. Los radios iónicos de $\mathrm{Ca}^{+2} \mathrm{y} \mathrm{Mg}^{+2}$ son 0,099 nm y 0,065 nm, respectivamente. Los cationes son fácilmente intercambiados por aquellos de la zeolita $\mathrm{Na}-\mathrm{X}(0,098 \mathrm{~nm})$. Las altas remociones de amonio y dureza principalmente, pueden ser explicadas por la gran disponibilidad de los sitios de adsorción intercambiables en el área superficial disponibles (Ríos et al, 2010).

La evaluación de la capacidad de adsorción de un lecho de zeolita en una columna de adsorción, mostró una capacidad de adsorción del adsorbente para conductividad y dureza de $q_{b}=2,523 \mathrm{mg} \cdot \mathrm{g}^{-1}$ y $\mathrm{q}_{\mathrm{b}}=$ $6,567 \mathrm{mg} \cdot \mathrm{g}^{-1}$, respectivamente (Tabla 2). De acuerdo a los experimentos de isotermas tipo batch, se encontró que se ajustan mejor al modelo de Langmuir, seguido del modelo de Freundlich. Con este ajuste 
se determinó la capacidad máxima de adsorción de dureza de la zeolita sintetizada, con un valor de 230 $\mathrm{mg} \cdot \mathrm{g}^{-1}$. Esto significa que la capacidad de las columnas empacadas es menor que en los experimentos tipo batch. En los procesos tipo batch, el equilibrio se alcanzó en una hora de contacto, mientras que el tiempo de contacto en las columnas fue mucho menor. Esto sugiere que la adsorción en la columna no se encuentra en estado de equilibrio (Cortés, 2007). La eficiencia fue de aproximadamente $5 \%$ de la capacidad del adsorbente para retener dureza en sistemas continuos. Al incrementarse el tiempo de contacto, que es función de la altura de la columna, se incrementa la capacidad de adsorción del lecho y por tanto, la eficiencia.

Para el caso de la conductividad con lecho de zeolita, la capacidad de adsorción $\left(q_{b}=2,523\right)$ comparada con la capacidad de adsorción de dureza, disminuye notablemente; esto sugiere que el tipo de contaminante catiónico tiene un efecto significativo sobre la capacidad de adsorción en sistemas continuos, respecto a la capacidad de cada columna; teniendo en cuenta que las condiciones de operación fueron las mismas en ambos experimentos.

Por lo tanto, el flujo utilizado en las columnas no proporciona el tiempo de contacto necesario para que los iones $\mathrm{Ca}^{+2}$ y $\mathrm{Mg}^{+2}$ se distribuyan a través de toda el área superficial del adsorbente. Es por esto, que los procesos tipo batch proporcionan una mejor interacción entre los iones y el adsorbente que los sistemas en columna (Costafreda, 2008) y por esta razón existe una disminución significativa de las capacidades de adsorción en las columnas.

\section{CONCLUSIONES}

La zeolita faujasita sintetizada presenta una buena capacidad de remoción de los cationes típicos de ARD. La dureza (iones $\mathrm{Ca}$ y $\mathrm{Mg}$ ) es la que presenta mayor remoción en comparación con la materia orgánica (DQO). Se atribuye esta remoción a la capacidad de intercambio catiónico y adsorción de estos iones hidratados en el poro de la zeolita, según su tamaño y afinidad. La zeolita ha demostrado tener potencial aplicación para la implementación de columnas de adsorción e intercambio que permitan la remoción de cationes de agua residual doméstica, para esto, es necesario garantizar un tiempo de contacto adecuado para alcanzar el equilibrio de adsorción en estos sistemas.

\section{AGRADECIMIENTOS}

Los autores agradecen a la Vicerrectoría de Investigaciones de la Universidad de la Amazonia y al CODEC\&T Caquetá por financiar éste proyecto aprobado en el marco de la convocatoria del CODEC\&T de grupos de investigación del año 2012.

Tabla 2. Capacidad de adsorción $\left(q_{b}\right)$ de iones (en términos de conductividad y dureza) sobre la Zeolita sintetizada a diferentes alturas del lecho con $\mathrm{Qv}=0,012$ L. $\mathrm{min}^{-1-}$

\begin{tabular}{|c|c|c|c|c|c|}
\hline Parámetro & $\begin{array}{c}\text { Altura de } \\
\text { lecho } h(\mathrm{~cm})\end{array}$ & $\begin{array}{c}\text { Masa de la } \\
\text { zeolita } m_{c}(g)\end{array}$ & $\begin{array}{l}\text { Volumen tra- } \\
\text { tado } V(\mathrm{~mL})\end{array}$ & $\begin{array}{c}\text { Concen- } \\
\text { tración inicial } \\
\text { Co }\left(\mathrm{mg} \cdot \mathrm{L}^{-1}\right)\end{array}$ & $\begin{array}{c}\text { Capacidad de } \\
\text { adsorción } q_{b} \\
\left(m g \cdot g^{-1}\right)\end{array}$ \\
\hline $\begin{array}{l}\text { Conductividad } \\
\left(\mu \mathrm{S} . \mathrm{cm}^{-1}\right)\end{array}$ & $\begin{array}{l}1,00 \\
5,00 \\
10,0\end{array}$ & $\begin{array}{c}2,35 \\
11,30 \\
23,42\end{array}$ & $\begin{array}{l}100,0 \\
100,0 \\
100,0\end{array}$ & $\begin{array}{l}538 \\
538 \\
538\end{array}$ & $\begin{array}{l}0,543 \\
1,654 \\
2,523\end{array}$ \\
\hline Dureza (mg. $\left.\mathrm{L}^{-1}\right)$ & $\begin{array}{l}1,00 \\
5,00 \\
10,0\end{array}$ & $\begin{array}{c}2,35 \\
11,30 \\
23,42\end{array}$ & $\begin{array}{l}100,0 \\
100,0 \\
100,0\end{array}$ & $\begin{array}{l}50 \\
50 \\
50\end{array}$ & $\begin{array}{l}0,983 \\
2,098 \\
6,567\end{array}$ \\
\hline
\end{tabular}




\section{REFERENCIAS}

1. Castaldi Paola, Santona Laura, Enzo Stefano, Pietro Melis. 2008. Sorption processes and XRD analysis of a natural zeolite exchanged with $\mathrm{Pb}^{2+}, \mathrm{Cd}^{2+}$ and $\mathrm{Zn}^{2+}$ cations. Journal of Hazardous Materials 156: 428-434.

2. Cortés M., R. 2007. Efecto de la modificación de una zeolita natural mexicana en la sorción de cadmio y 4-clorofenol. Tesis doctoral. Facultad de ingeniería. Centro interamericano de recursos del agua. Universidad autónoma del estado de México.

3. Costafreda M, Jorge L. 2008. Geología, caracterización y aplicaciones de las rocas zeolíticas del complejo volcánico de Cabo de Gata (Almería). Tesis Doctoral. Universidad Politécnica de Madrid. Escuela Técnica Superior de Ingenieros de Minas.

4. Dussan A, Otálora B. J. H., Chica B. R, Bonilla R. N. C, Otálora B. D. M. 2012. Síntesis y estudio de las propiedades estructurales de zeolitas crecidas a partir del sistema $\mathrm{NaOH}+\mathrm{H}_{2} \mathrm{O}+\mathrm{A}^{*}$ $\left(A^{*}=\mathrm{SiO}_{2}, A l\right)$ en un medio alcalino $(\mathrm{pH}>10)$. Avances investigación en ingeniería 9(2): 53-59.

5. Fu, F. y Wang. Q. 2011. Removal of heavy metal ions from wastewaters. Journal of Environmental Management. 92(3): 407-418.

6. Manrique-Losada Lis, Marlon Pelaez-Rodríguez. 2010. Evaluación de la Calidad del Agua de la Quebrada la Perdiz en el Área Urbana de Florencia (Caquetá-Colombia). Momentos de Ciencia 7 (2):118-126.

7. Mekatel, H., Amokrane, S., Benturki, A. \& Nibou D. 2012. Treatment of Polluted Aqueous Solutions by $\mathrm{Ni}^{2+}, \mathrm{Pb}^{2+}, \mathrm{Zn}^{2+}, \mathrm{Cr}^{+6}, \mathrm{Cd}^{+2}$ and $\mathrm{Co}^{+2}$ lons by Ion Exchange Process Using Faujasite Zeolite. Procedia Engineering 33: 52 - 57.
8. Moulin, B, Oliviero, L., Maugé F., Groust, J-F., Krafft J.-M., Costentin, G., Massiani P. 2008. Probing the strength, concentration and environment of basic sites in zeolites by IR spectroscopy. Zeolites and Related Materials: Trends, Targets and Challenges Proceedings of 4th International FEZA.

9. Nibou D., Amokrane S. \& Lebaili N. 2010. Use of $\mathrm{NaX}$ porous materials in the recovery of iron ions. Desalination 250: 459-462.

10. Nibou D., Mekatel H., Amokrane S., Barkat M., M. Trari. 2010b. Adsorption of Zn2+ ions onto $\mathrm{NaA}$ and $\mathrm{NaX}$ zeolites: Kinetic, equilibrium and thermodynamic studies.. Journal of Hazardous Materials 173: 637-646.

11. Ríos, C. A., Williams, C. D., Roberts C. L. 2010. Zeolitas a base de cenizas volantes del Reino Unido como adsorbentes para la remoción de metales pesados y amonio a partir de soluciones contaminadas artificialmente. Ingeniería y Competitividad, Volumen 12(1): 57-71.

12. Roldán, P. G. \& J. J. Ramírez. 2008. Fundamentos de Limnología Neotropical. Segunda Edición. Editorial Universidad de Antioquia. Medellín.

13. Rozic, M., Cerjan-Stefanovic, S., Kurajica S., Vancina, V., Hodzic, E. 2000. Ammoniacal nitrogen removal from water by treatment with clays and zeolites, Water Research 34: 3675-3681.

14. Sprynskyy M., Buszewski B., Terzyk A.P., Namie'snik J., 2006. Study of the selection mechanism of heavy metal $\left(\mathrm{Pb}^{2+}, \mathrm{Cu}^{2+}, \mathrm{Ni}^{2+}\right.$, and $\mathrm{Cd}^{2+}$ ) adsorption on clinoptilolite, J. Colloid Interface Sci. 304. 21-28.

15. Wang, S. \& Peng, Y. 2010. Natural zeolites as effective adsorbents in water and wastewater treatment. Chemical Engineering Journal 156: 11-24. 